\title{
Das verflixte erste Jahr
}

\section{Adrian Ritter}

Freier Journalist

\author{
Das erste Jahr der Assistenzarztzeit ist bisweilen ein Sprung ins kalte Wasser. Das \\ diesjährige Symposium für ärztliche Weiterbildner widmete sich den Problemen \\ des ersten Weiterbildungsjahres und diskutierte Lösungen.
}

Der Einstieg in die Zeit der Weiterbildung nach dem Medizinstudium ist nicht immer einfach. Das wurde am diesjährigen Symposium für ärztliche Weiterbildner am 19. Februar im UniversitätsSpital Zürich klar. Unter dem Motto «Mit den richtigen Lernzielen zum Erfolg» widmete sich das Symposium spezifisch dem ersten Weiterbildungsjahr.

Jürg Hodler, ärztlicher Direktor des UniversitätsSpitals Zürich, zeigte mögliche Herausforderungen und Schwierigkeiten dabei auf: zu fordernde und abwesende Vorgesetzte, eine fehlende Laufbahnplanung und beim Weiterzubildenden Fehleinschätzungen der eigenen Fähigkeiten, die drückende Last der Verantwortung und die Angst, nicht zu genügen.

Diese möglichen Probleme schaden nicht nur den angehenden Ärztinnen und Ärzten, wie Jürg Hodler sagte. Auch für die Arbeitgeber hätten sie Nachteile, etwa ungeplante Kündigungen und Imageschäden - kein vernachlässigbarer Faktor in Zeiten des Ärztemangels. Das UniversitätsSpital Zürich versuche die Assistenzarztzeit unter anderem mit Weiterbildungsverantwortlichen, einem Laufbahnmodell und unterstützenden Veranstaltungen für Weiterzubildende hilfreich zu gestalten.

\section{Première année difficile}

Des supérieurs trop exigeants ou absents, le manque de planification de carrière et la crainte de ne pas être à la hauteur. Au terme des études de médecine, il n'est pas toujours facile de débuter sa période d'assistanat. C'est ce qu'a démontré le symposium pour les responsables de la formation postgraduée qui a eu lieu le 19 février dernier à l'Hôpital universitaire de Zurich sous le titre "La première année de formation postgraduée». Lors de cette manifestation, des solutions créatives ont été proposées quant à la manière d'appréhender le passage des études de médecine à l'assistanat. En Suisse romande, les hôpitaux sont réunis en réseau pour organiser la formation postgraduée. A l'université de Zurich, I'«option spécifique en psychiatrie» sert déjà durant les études de médecine de tremplin vers la première année de formation postgraduée. Le concept des «Entrustable Professional Activities» (EPAs) a également suscité I'intérêt du public. Ce concept permet aux médecins en formation d'assumer progressivement de plus en plus de compétences selon leur degré d'autonomie.
Jede anerkannte Weiterbildungsstätte für Ärztinnen und Ärzte muss über ein Weiterbildungskonzept verfügen, wie Werner Bauer, Präsident des Schweizerischen Instituts für ärztliche Weiter- und Fortbildung (SIWF), aufzeigte. In diesen Konzepten müsse auch die Einführungszeit von Assistenzärztinnen und -ärzten klar strukturiert werden. Werner Bauer stellt bei Visitationen in den Ausbildungsstätten denn auch fest, dass der Einstieg ins erste Weiterbildungsjahr bewusster gestaltet wird als früher.

\section{Sich neu beweisen}

Klaus Grätz, Delegierter für ärztliche Fortbildung und Laufbahnentwicklung am UniversitätsSpital Zürich, liess im Gespräch mit Christian Fankhauser und

Der Einstieg ins erste Weiterbildungsjahr wird bewusster gestaltet als früher.

Alexandra Hubler zwei Assistenzärzte aus der Praxis berichten. Christian Fankhauser hat die Einführung in jeder der vier Kliniken seines bisherigen Arbeitslebens anders erlebt. Als hilfreich erwiesen sich für ihn unter anderem Guidelines und Checklisten für die Einarbeitung. Eher frustrierend sei die Erfahrung, eine bestimmte Tätigkeit am nächsten Arbeitsort plötzlich nicht mehr ausführen zu dürfen. "Man muss sich an jedem Spital wieder neu beweisen», hat auch Alexandra Hubler die Erfahrung gemacht.

\section{Mit EPAs in die Zukunft?}

Wann darf ein Arzt oder eine Ärztin in Weiterbildung eine bestimmte Aufgabe zusätzlich übernehmen? Darüber werde bisweilen nach eher undurchsichtigen Kriterien am Tisch eines Vorgesetzten beschlossen, hat Pascal Berberat die Erfahrung gemacht. Der Professor für Medizindidaktik, medizinische Lehrentwicklung und Bildungsforschung an der Technischen Universität München kritisierte in seinem Referat ein solch intransparentes und wenig standardisiertes Verfahren. 


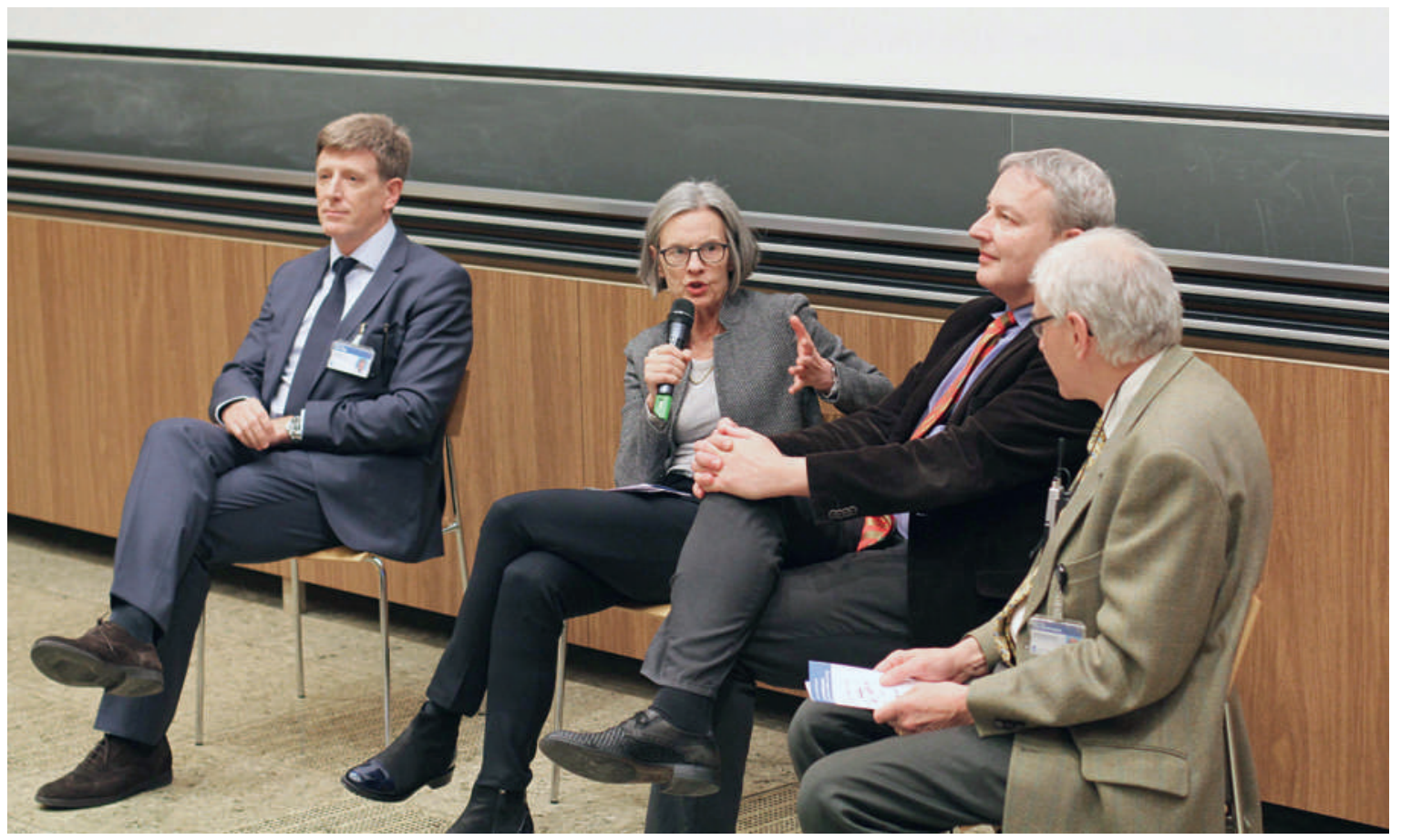

Barbara Federspiel, Chefärztin an der Klinik für Innere Medizin des See-Spitals Horgen, diskutierte angeregt mit Luca Regli, Klinikdirektor Neurochirurgie am UniversitätsSpital Zürich (links), Stefan Wildi, Chefarzt der Chirurgischen Klinik am Zürcher Waidspital (zweiter von rechts), und Hans-Ulrich Bucher, Organisator des Symposiums (erster von rechts).

Berberat präsentierte die «Entrustable Professional Activities» (EPAs), die sich explizit vom klinischen Alltag aus definieren. Eine EPA ist eine Arbeitseinheit, die dem Weiterzubildenden übertragen werden kann unter der Voraussetzung, dass er ausreichend Kompetenzen zu ihrer Erfüllung gezeigt hat. Entscheidend ist der Grad der Selbständigkeit, wobei fünf Leistungsniveaus unterschieden werden: Der Arzt oder die Ärztin in Weiterbildung kann eine bestimmte Arbeit (1) noch nicht durchführen, (2) unter enger Begleitung durchführen, (3) mit der Möglichkeit, Unterstützung anzufordern, durchführen oder (4) selbständig durchführen. Ab diesem Stadium könne von "Anvertrauen" (entrust) gesprochen werden, so Berberat. Auf Stufe (5) kann der Arzt oder die Ärztin in Weiterbildung andere Personen bereits selber bei der Durchführung der Tätigkeit betreuen.

Die Weiterbildung ist gemäss diesem System nicht fix nach einer bestimmten Anzahl Jahre abgeschlossen, sondern dann, wenn jemand bei allen nötigen EPAs die Stufe (4) erreicht hat. Derzeit werde in der Fachwelt diskutiert, wie viele EPAs sinnvollerweise für einen Facharzttitel zu definieren seien - «schätzungsweise 15 bis 20 ", so Berberat.

Schritt für Schritt mehr Kompetenzen übernehmen, so die Grundidee des EPA-Systems. Wie aber kann eingeschätzt werden, auf welcher Stufe sich jemand befin- det? Für Pascal Berberat sind dazu nicht unzählige Formulare nötig. Gewisse Kernaktivitäten müssten direkt beobachtet und dokumentiert werden (etwa mit miniCEX, DOPS). Hauptsächlich aber sollte die Einschätzung im Austausch verschiedener Personen erfolgen etwa in einer monatlichen "Oberarztrunde». Es gelte, im Gespräch einen Konsens zu finden, wie der Weiterzubildende einzustufen sei - allenfalls auch unter Einbezug der Pflegenden und Patienten, so Berberat.

\section{Wann dürfen weiterzubildende Ärzte eine bestimmte Aufgabe zusätzlich übernehmen?}

Zentral sei dann die Rückmeldung an die Weiterzubildenden. Zurzeit wird das EPA-System gemäss Berberat weltweit diskutiert. Insbesondere in den angelsächsischen Ländern seien Pilotprojekte in Arbeit.

Hans Ulrich Bucher, Beauftragter für ärztliche Weiterbildung am UniversitätsSpital Zürich und Organisator des Symposiums, informierte über den Stand der Diskussion zu EPAs in der Schweiz. Im Bereich Weiterbildung befinde sich die Diskussion noch ganz am Anfang - am weitesten fortgeschritten in der Anästhesiologie. Eine Expertengruppe schlage das EPA-System aber für das Medizinstudium vor - eine Idee, die sich derzeit in der Vernehmlassung bei den medizinischen Fakultäten befinde. 


\section{Sprungbrett in die Weiterbildung}

Eine besondere Form, den Einstieg in die Assistenzarztzeit einfacher zu gestalten, präsentierte am Symposium Ulrich Schnyder, Klinikdirektor Psychiatrie und Psychotherapie am UniversitätsSpital Zürich. Seit 2011 können an der Universität Zürich Studierende der Medizin einen «Studienschwerpunkt Psychiatrie» wählen. Wer schon früh weiss, dass er sich für die Fachrichtung Psychiatrie interessiert, kann mehrere Module im Bereich Psychiatrie buchen und dort auch seine Masterarbeit schreiben. Zusätzlich nehmen die Studierenden an einem Mentoringprogramm teil. Der Vorteil: «Bereits das Studium wird zu einem Sprungbrett ins erste Weiterbildungsjahr», so Ulrich Schnyder. Er ist überzeugt, dass die Absolvierenden für Arbeitgeber wie Psychiatrische Kliniken attraktiv sind. Das Konzept müsse nicht auf die Psychiatrie beschränkt bleiben, sondern könnte auch auf andere Fachbereiche übertragen werden, so Schnyder. Hilfreich können im ersten Weiterbildungsjahr auch Formen des E-Learning und Simulationen sein, wie Adrian Marty als Leiter des Simulationszentrums des UniversitätsSpitals Zürich aufzeigte. «Wenn es eine entsprechende Simulation gibt, sollte diese unbedingt genutzt werden, bevor ein Assistenzarzt im ersten Weiterbildungsjahr ohne Erfahrung direkt einen Patienten behandelt», so Marty.

\section{Weiterbildung im Verbund}

Nathalie Koch, Leiterin der ärztlichen Weiterbildung und Laufbahnplanung am Centre Hospitalier Universitaire Vaudois (CHUV) in Lausanne, stellte in ihrem Referat die Weiterbildungsnetzwerke der Romandie vor. Dabei organisieren die Spitäler der französischsprachigen Schweiz die Weiterbildung gemeinsam von der Rekrutierung über die Strukturierung bis zur Evaluation. Inzwischen existieren bereits Weiterbil- dungsnetzwerke in den Bereichen Anästhesie, Gynäkologie, Pädiatrie und Orthopädie - weitere sind in der Chirurgie und im Bereich HNO in Entwicklung.

\section{Ein gutes Klima schaffen}

Wie auch immer das erste Weiterbildungsjahr gestaltet und organisiert ist, gilt es doch zu bedenken: «Weiterzubildende sind nicht nur 'workers', sondern auch 'learners'», wie es Klaus Grätz vom UniversitätsSpital Zürich ausdrückte. Um eine gute Betreuung der Lernenden zu gewährleisten, sollten deren Betreuer durch nicht-ärztliches Personal (Physician Assistants) vermehrt entlastet werden, sagte Luca Regli, Klinikdirektor Neurochirurgie am UniversitätsSpital Zürich. Bar-

\section{Betreuer von weiterzubildenden Ärzten sollen vermehrt durch nicht-ärztliches Personal entlastet werden.}

bara Federspiel, Chefärztin an der Klinik für Innere Medizin des See-Spitals Horgen, schlug vor, als Vorbereitung auf die Assistenzarztzeit bereits das Wahlstudienjahr verbindlicher und strukturierter zu gestalten. Für die Weiterzubildenden gelte es, vor allem auch ein gutes Klima und einen geschützten Rahmen zu schaffen, beschrieb Stefan Wildi, Chefarzt der Chirurgischen Klinik am Zürcher Waidspital, die Aufgabe der Ausbildungsstätten. Einig waren sich Referierende und Votanten aus dem Publikum des Symposiums, dass die bereits ausgebildeten Ärztinnen und Ärzte wichtige Vorbilder für den Nachwuchs sind.

Bildnachweis

Foto von Adrian Ritter

Die Podcasts und Tagungsunterlagen zum Symposium sind auf www.usz.ch/bl zu finden. Das nächste Symposium für ärztliche Weiterbildner findet am 10. Februar 2017 statt. 